\title{
Resolving the dusty torus and the mystery surrounding LMC red supergiant WOH G64
}

\author{
Keiichi Ohnaka ${ }^{1}$, Thomas Driebe ${ }^{1}$, Karl-Heinz Hofmann ${ }^{1}$, \\ Gerd Weigelt $^{1}$ and Markus Wittkowski ${ }^{2}$ \\ ${ }^{1}$ Max-Planck-Institut für Radioastronomie, Auf dem Hügel 69, 53121 Bonn, Germany \\ email: kohnaka@mpifr-bonn.mpg.de \\ ${ }^{2}$ European Southern Observatory, Karl-Schwarzschild-Str. 2, 85748 Garching, Germany
}

\begin{abstract}
We present mid-IR long-baseline interferometric observations of the red supergiant WOH G64 in the Large Magellanic Cloud with MIDI at the ESO's Very Large Telescope Interferometer (VLTI). Our MIDI observations of WOH G64 are the first VLTI observations to spatially resolve an individual stellar source in an extragalactic system. Our 2-D radiative transfer modeling reveals the presence of a geometrically and optically thick torus seen nearly pole-on. This model brings WOH G64 in much better agreement with the current evolutionary tracks for a $25 \mathrm{M}_{\odot}$ star — about a half of the previous estimate of $40 \mathrm{M}_{\odot}$ - and solves the serious discrepancy between theory and observation which existed for this object.
\end{abstract}

Keywords. techniques: interferometric, infrared: stars, circumstellar matter, stars: evolution, stars: late-type, stars: mass loss, supergiants, Magellanic Clouds

\section{Introduction}

Red supergiants (RSGs) in the Large and Small Magellanic Clouds (LMC and SMC, respectively) provide an excellent opportunity to observationally test the current stellar evolution theory for massive $\left(\geqslant 8 \mathrm{M}_{\odot}\right)$ stars. Another advantage of studying RSGs in the LMC and SMC is that we can probe metallicity effects on the mass loss.

WOH G64 is a luminous RSG in the LMC, surrounded by an optically thick dust envelope with the $10 \mu \mathrm{m}$ silicate feature seen in self-absorption. The strength of the TiO bands suggests spectral types of M5-M7 (Elias et al. 1986; van Loon et al. 2005), which translate into $T_{\text {eff }}=3200-3400 \mathrm{~K}$. The luminosities of $5-6 \times 10^{5} \mathrm{~L}_{\odot}$ estimated by these authors assuming spherical shells correspond to an initial mass of $\sim 40 \mathrm{M}_{\odot}$. However, this $\mathrm{T}_{\text {eff }}$ is too low for the current evolutionary tracks for a $40 \mathrm{M}_{\odot}$ star (discrepancy in temperature $\approx 3000 \mathrm{~K}$ ! - see the box in Fig. 1 ). The above authors note that the low obscuration in the near-IR/visible, despite the huge mid-/far-IR excess (Fig. 3a), may indicate the possible presence of a disk or torus. Such deviation from spherical symmetry affects the luminosity estimate. To examine this possibility, we carried out mid-IR highspatial resolution observations of WOH G64 with VLTI/MIDI.

\section{VLTI/MIDI Observations}

MIDI is a $10 \mu \mathrm{m}$ interferometric instrument which combines two beams from $8.2-\mathrm{m}$ Unit Telescopes (UTs) or movable 1.8-m Auxiliary Telescopes (ATs). MIDI measures the "visibility amplitude" (= Fourier amplitude of the object's intensity distribution), which contains information about the object's size and shape. We observed WOH G64 in 2005 and 2007 with the UT3-UT4-62m baseline with a spatial resolution of $~ 10$ mas. 


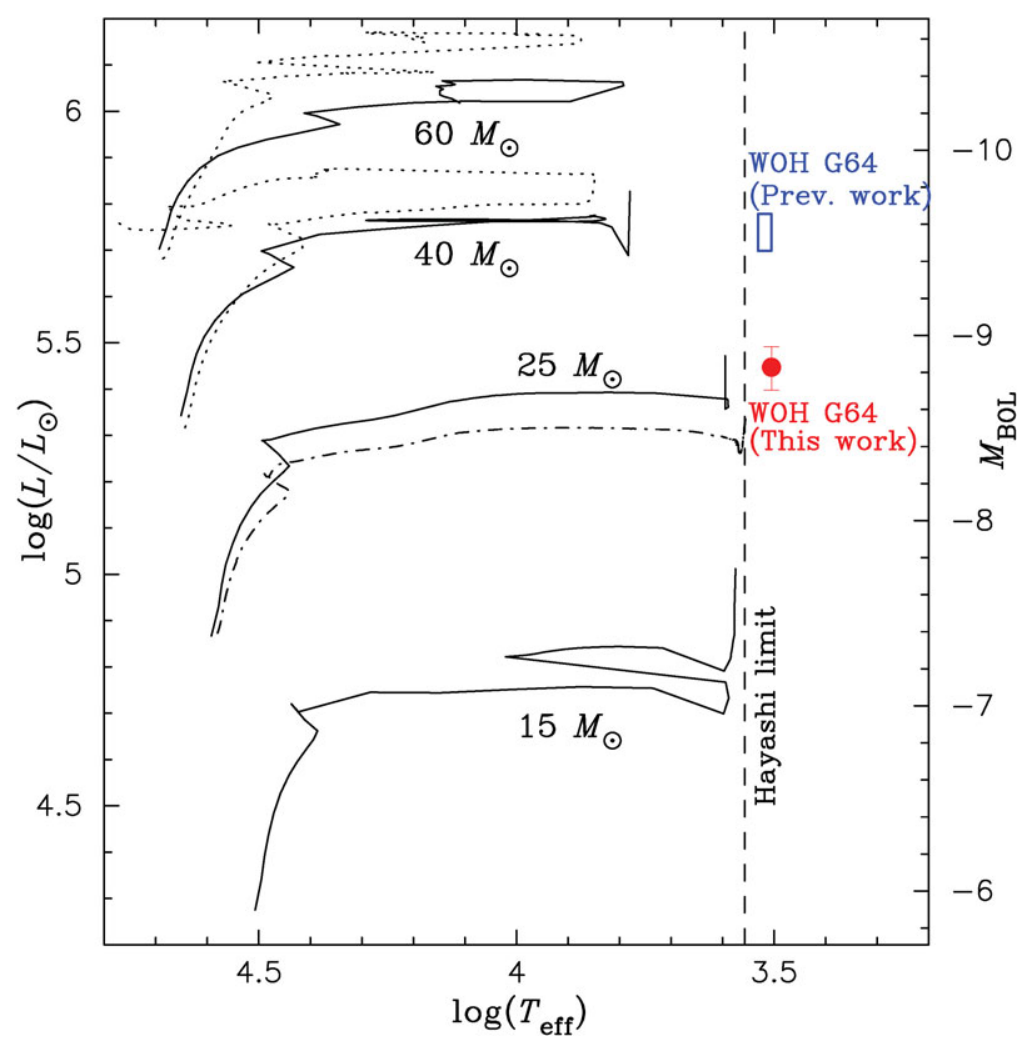

Figure 1. H-R diagram with theoretical evolutionary tracks with $Z=0.008$ (solid lines: Schaerer et al. 1993; dotted lines: Meynet \& Maeder 2005) and that newly calculated with $Z=0.01$ by T. Driebe (dashed-dotted line). The box and circle represent the observationally derived locations of WOH G64. The dashed line represents the Hayashi limit.

We measured visibilities at four position angles differing by $\sim 60^{\circ}$ but at almost the same baseline length $(57-62 \mathrm{~m})$. Interferometric fringes were spectrally dispersed with $\lambda / \Delta \lambda \approx 30$ between 8 and $13 \mu \mathrm{m}$ (see Fig. 2). We found no significant temporal variation in the $N$-band spectra between the two epochs (also in agreement with the Spitzer/IRS data taken in 2005), and so we merged the data taken in 2005 and 2007.

\section{Observational results: interferometry and spectroscopy}

Figure 3c shows the $N$-band visibilities observed toward WOH G64. This is the first $V L T I$ observations to spatially resolve an individual star in an extragalactic system. We fitted the observed visibilities with uniform-disks, and the resulting angular diameters are shown in Fig. 3d. The uniform-disk diameters increase from $\sim 15$ to 23 mas between 8 and $10 \mu \mathrm{m}$ and is roughly constant above $10 \mu \mathrm{m}$. As Figs. $3 \mathrm{c}$ and $3 \mathrm{~d}$ show, the visibilities and angular diameters measured at four position angles do not show a noticeable difference, suggesting that the object appears nearly centrosymmetric.

We also identified the $\mathrm{H}_{2} \mathrm{O}$ absorption features at 2.7 and $6 \mu \mathrm{m}$ in the spectra obtained with the Short Wavelength Spectrometer (SWS) and PHOT-S onboard the Infrared Space Observatory and the InfraRed Spectrometer (IRS) onboard the Spitzer Space Telescope (Fig. 3b). The $2.7 \mu \mathrm{m}$ feature ( $\nu_{1}$ and $\nu_{3}$ fundamental bands) originates in the photosphere and/or the extended molecular layers (so-called MOLsphere), while the $6 \mu \mathrm{m}$ feature ( $\nu_{2}$ fundamental bands) is likely to be of circumstellar origin. 


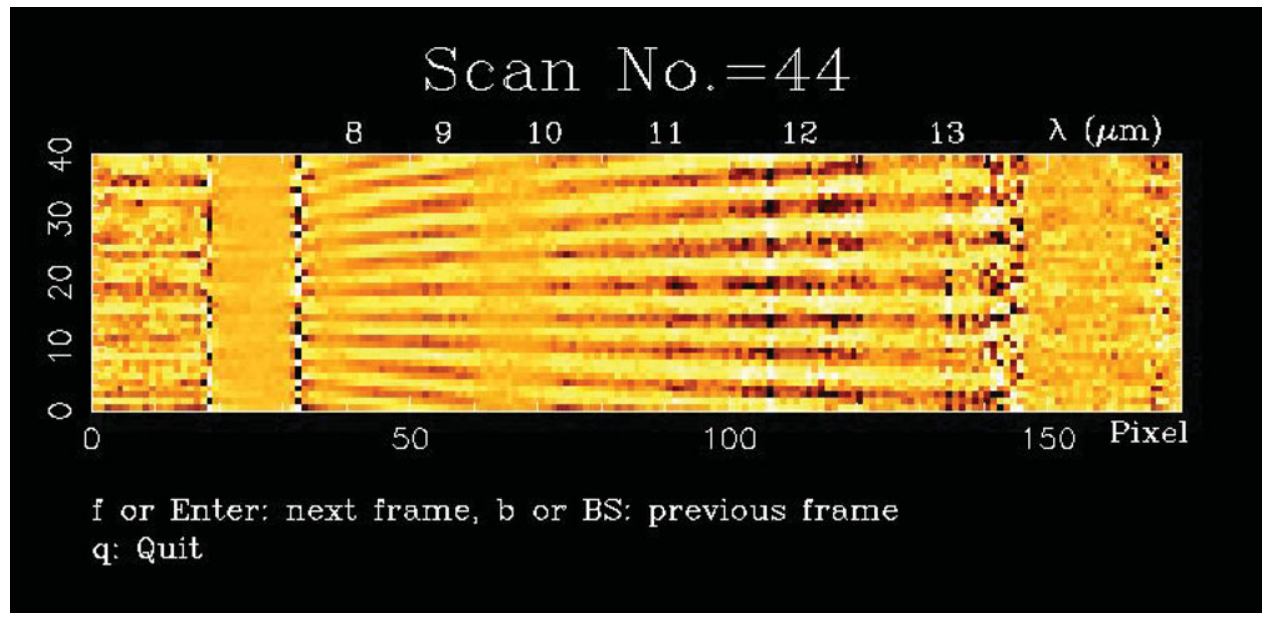

Figure 2. Spectrally dispersed fringes observed for WOH G64 with MIDI. These fringes were visualized by stacking 40 frames taken during one scan in the vertical direction. Each row corresponds to one frame.

\section{2-D radiative transfer modeling}

We performed 2-D radiative transfer modeling to characterize the dust envelope around WOH G64, using our Monte Carlo code (see Ohnaka et al. 2008 for details).

The observed $N$-band visibilities and SED can be reproduced by an optically and geometrically thick silicate torus model viewed close to pole-on (inset of Fig. 3a). This pole-on model can explain the low obscuration in the near-IR/visible and the absence of position angle dependence of the visibilities (solid lines in Fig. 3). The derived dust torus parameters are as follows: $\tau_{\mathrm{V}}=30 \pm 5$ (optical depth at $0.55 \mu \mathrm{m}$ in the equatorial plane), $\tau_{\mathrm{V}}=9 \pm 2$ (optical depth along the line of sight), inner boundary radius $=15 \pm 5 \mathrm{R}_{\star}$ $\left(R_{\star}=1730 \mathrm{R}_{\odot}, \rho \propto r^{-2}\right.$ was assumed), torus half-opening angle $=60 \pm 10^{\circ}$. The derived luminosity, $\sim 2.8 \times 10^{5} \mathrm{~L}_{\odot}$, is about a half of the previous estimates based on spherical models. This is because we look into the torus from nearly pole-on. Radiation escapes preferentially through the cavity (i.e., toward us), and the luminosity is overestimated when derived assuming spherical symmetry.

The new, lower luminosity brings the location of WOH G64 on the H-R diagram in much better agreement with theoretical evolutionary tracks for a $25 \mathrm{M}_{\odot}$ star (filled circle in Fig. 1). We also note that WOH G64 lies very close to or even beyond the Hayashi limit (dashed line in Fig. 1), which implies that this object may be experiencing unstable, violent mass loss. The derived total envelope mass, 3-9 $\mathrm{M}_{\odot}$, represents a considerable fraction of its initial mass, which is consistent with this picture.

\section{Conclusion}

We have spatially resolved the circumstellar environment of the red supergiant WOH G64 in the LMC for the first time. Our 2-D radiative transfer modeling shows the presence of an optically and geometrically thick torus viewed close to pole-on and brings the location of WOH G64 in much better agreement with the current stellar evolution theory. Now, MIDI observations with shorter and longer baselines are indispensable for obtaining tighter constraints on the torus inner boundary radius, which is crucial for understanding metallicity effects on the dust formation and mass loss in RSGs. 

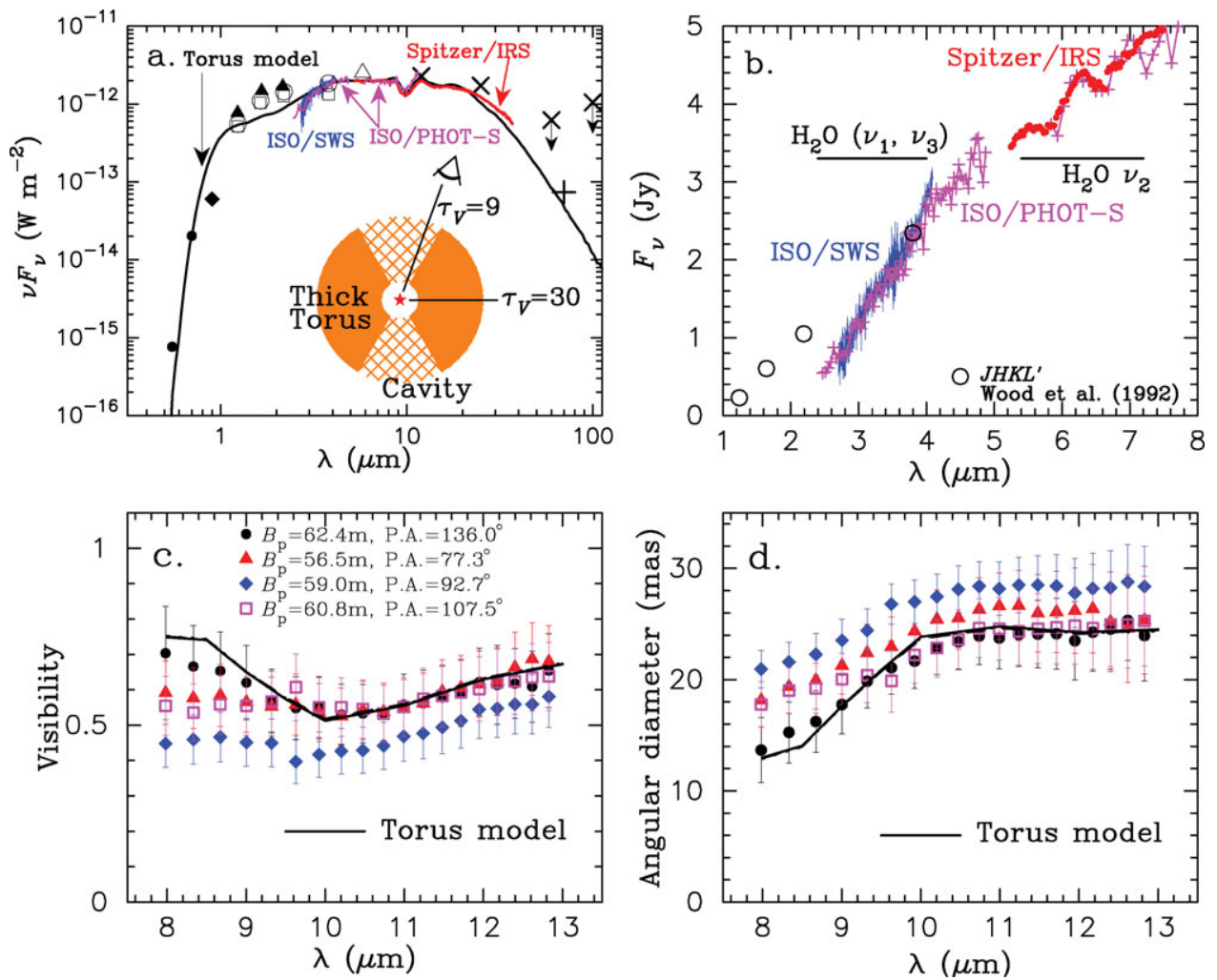

Figure 3. a: SED. filled circles: MACHO (Alcock et al. 2000), filled diamond: ASAS (Pojmański 2002, 2003; Pojmański \& Maciejewski 2004, 2005; Pojmański et al. 2005), filled triangles: 2MASS (Cutri et al. 2003), open circles: Wood et al. (1992), open squares: Whitelock et al. (2003), open triangle: SAGE (Meixner et al. 2006), ×: IRAS, +: Spitzer/MIPS. b: $\mathrm{H}_{2} \mathrm{O}$ absorption between 2 and $8 \mu \mathrm{m}$. c: $N$-band visibilities observed with MIDI and the torus model. The projected baseline lengths $\left(B_{\mathrm{p}}\right)$ and the position angles (P.A.) are also given. d: $N$-band angular diameters observed at four position angles and the torus model.

\section{References}

Alcock, C., Allsman, R., Alves, D., et al. 2000, ApJ, 542, 281

Cutri, R. M., Skrutskie, M. F., van Dyk, S., et al. 2003, The IRSA 2MASS All-Sky Catalog of Point Sources, NASA/IPAC Infrared Science Archive

Elias, J. H., Frogel, J. A., \& Schwering, P. B. 1986, ApJ, 302, 675

Meynet, G. \& Maeder, A. 2005, A\&A, 429, 581

Meixner, M., Gordon, K. D., Indebetouw, R., et al. 2006, AJ, 132, 2268

Ohnaka, K., Driebe, T., Hofmann, K. -H., Weigelt, G., \& Wittkowski, M. 2008, A 6 A, 484, 371

Pojmański G. 2002, AcA, 52, 397

Pojmański G. 2003, AcA, 53, 341

Pojmański G. \& Maciejewski, G. 2004, AcA, 54, 153

Pojmański G. \& Maciejewski, G. 2005, AcA, 55, 97

Pojmański G., Pilecki, B, \& Szczygieł, D. 2005, AcA, 55, 275

Schaerer, D., Meynet, G., Maeder, A., \& Schaller, G. 1993, A\&\&AS, 98, 523

van Loon, J. Th., Cioni, M. -R. L., Zijlstra, A. A., \& Loup, C. 2005, A\&AA, 438, 273

Whitelock, P., Feast, M. W., van Loon, J. Th., \& Zijlstra, A. A. 2003, MNRAS, 342, 86

Wood, P. R., Whiteoak, J. B., Hughes, S. M. G., et al. 1992, ApJ, 397, 552 


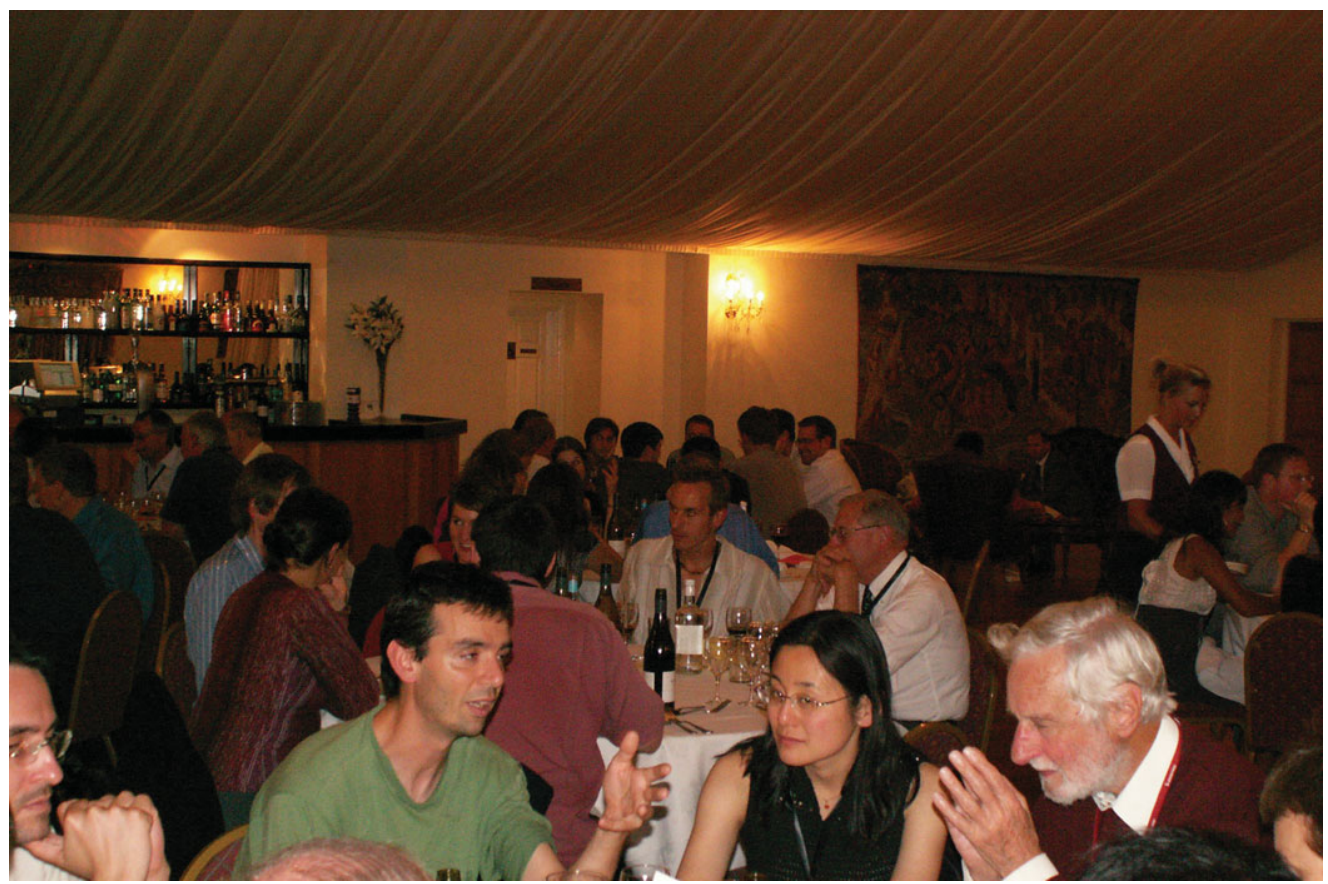

At the conference dinner inside Wrenbury Hall.

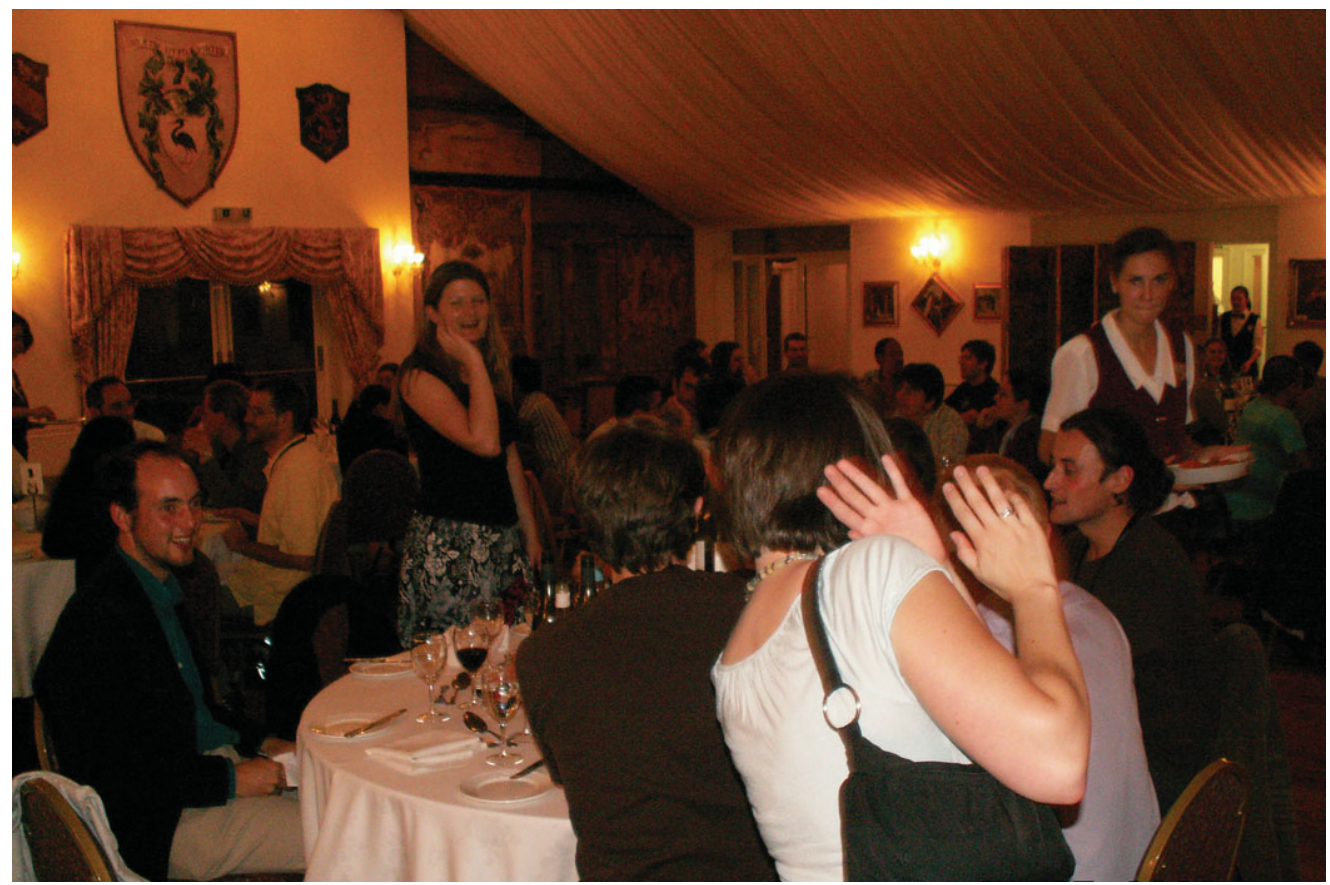

Getting quite lively... 